C2012, Elsevier. Licensed under the Creative Commons Attribution-NonCommercialNoDerivatives 4.0 International http://creativecommons.org/about/downloads 


\title{
A Review on Vision Techniques applied to Human Behaviour Analysis for Ambient-Assisted Living
}

\author{
Alexandros André Chaaraoui, Pau Climent-Pérez, Francisco \\ Flórez-Revuelta* \\ Department of Computing Technology, University of Alicante, P.O. Box 99, E-03080, \\ Alicante, Spain
}

\begin{abstract}
Human Behaviour Analysis (HBA) is more and more being of interest for Computer Vision and Artificial Intelligence researchers. Its main application areas, like Video Surveillance and Ambient-Assisted Living (AAL), have been in great demand in recent years. This paper provides a review on HBA for AAL and ageing in place purposes focusing specially on vision techniques. First, a clearly defined taxonomy is presented in order to classify the reviewed works, which are consequently presented following a bottom-up abstraction and complexity order. At the motion level, pose and gaze estimation as well as basic human movement recognition are covered. Next, the mainly used action and activity recognition approaches are presented with examples of recent research works. Increasing the degree of semantics and the time interval involved in the HBA, finally the behaviour level is reached. Furthermore, useful tools and datasets are analysed in order to provide help for initiating projects.
\end{abstract}

Keywords: Human behavior, ambient-assisted living, computer vision, motion analysis, action recognition, activity recognition, activities of daily living (ADLs)

*Corresponding author: Francisco Flórez-Revuelta, Department of Computing Technology, University of Alicante, P.O. Box 99, E-03080, Alicante, Spain. Phone: +34 965903681, Fax: +34 965909643

Email addresses: alexandros@dtic.ua.es (Alexandros André Chaaraoui), pcliment@dtic.ua.es (Pau Climent-Pérez), florez@dtic.ua.es (Francisco Flórez-Revuelta) 


\section{Introduction}

Human Behaviour Analysis - and Understanding- (HBA, HBU) involves a wide range of investigation fields from motion detection and background extraction to expert systems and high-level abstraction behaviour models. This paper targets two purposes: On the one hand, researchers need to categorise existing works assuming a common taxonomy and a clear differentiation basis. On the other hand, as the application areas of these fields grow constantly; stable areas, like Video Surveillance, are covered thoroughly; while other more recent areas, like Ambient-Assisted Living (AAL) and ageing in place at smart home scenarios, present a lack of unifying works and recent state-of-the-art reviews. This makes initiation in these areas difficult, also because of the involvement of a wide variety of pure research areas from Artificial Intelligence to Natural Language Processing.

For this reason, this paper deals with the state-of-the-art of HBA/HBU from an Ambient Intelligence (AmI) point of view, focusing especially on indoor scenarios and techniques which are designed for AAL purposes. This way, recognition of Activities of Daily Living (ADLs) covers the main interest of this paper. Nevertheless, it is necessary to first face a classification of HBA levels, and to deal with all the necessary previous tasks.

To avoid the common difficulties present in vision-based systems (such as occlusions, view-dependent features, lightning conditions, etc.), occasionally these systems are enhanced with other sensors; mostly binary sensors and RFID labels. Therefore, although vision will be focused on mainly, other complementary sensors involved will be discussed briefly too.

The remainder of this paper is organised as follows: Section 2 goes through taxonomies which are applied by other authors and presents an abstraction, degree of semantics and time-oriented classification which is used in the rest of the paper. Section 3 deals with the lowest level, i.e. pose, gaze and motion estimation. These elements are used as action primitives in Section 4 where human actions are recognised based on video data and other sensor data fusion (RFID tags, accelerometers, etc.). Section 5 focuses on activity recognition methods which are of special interest in AAL: ADLs in indoor environments, like cooking and grooming, are recognised with different approaches detailed in that section. Finally, Section 6 deals with behaviour recognition methods that establish the highest degree of abstraction. Section 7 summarises some of the most used datasets and tools in the reviewed works that are available. 


\section{HBA Taxonomies}

In this section, different Human Behaviour Analysis taxonomies from some of the most recent and relevant research works are discussed in order to point out differences and converge at a well-defined classification of the works analysed in following sections.

Moeslund et al. [72] defined an action taxonomy which has been adopted in later works and subsequent surveys. From lower to higher degree of abstraction three levels are defined:

- Basic motion recognition derives in so called action or motor primitives representing the atomic entities out of which actions are built. Therefore, as stated in [88], an action primitive is an atomic movement that can be described at the limb level.

- A set of different or repetitive action primitives make up an action.

- Involving a larger scale of events, the context of the environment and the interacting objects or humans it is possible to recognise the actual activity.

This way, when making a cup of tea, single movements of arms and hands would be action primitives; placing the kettle on the stove or grabbing a cup from the cupboard would be actions; and finally, the whole process would make up an activity as different actions and interaction with several objects are involved.

Although this taxonomy is clearly defined and quite often referenced in HBA-related papers, most researchers use their own taxonomy, as usefulness depends on research goals and application areas. Since this classification is particularly focused on actions, it is difficult to adapt to higher level approaches, where the main targets are ADLs and behaviour analysis.

In [129], activities are defined as the combination of actions and objects. Whereas actions are recognised by a set of verbs, objects or places are recognised by a set of nouns which are targets of actions. Instead of recognizing the actions, object recognition is tackled in order to infer human activities. Turaga et al. [118] distinguish between actions and activities by defining that activities involve coordinated actions among a small number of humans.

Regarding behaviour analysis, Ji et al. [45] define behaviours as human motion patterns involving high-level description of actions and interactions. In contrast to [72], dependence on the context of the environment, objects 


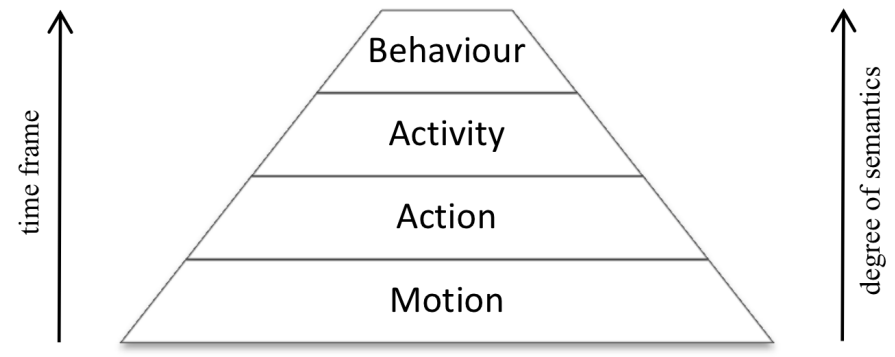

Figure 1: Human Behaviour Analysis tasks - Classification.

and human interaction are taken into account at the behaviour level. In [73], behaviours are understood as patterns in a sequence of observations of activities or events. Activities such as cooking, eating, watching TV or no detectable activities; and events from the environment, emitted by binary sensors installed in smart homes, enable to recognise repeatable patterns and detect anomalies.

In this paper, HBA tasks are classified into motion, action, activity or behaviour levels regarding the degree of semantics and the amount of time involved in the analysis. Therefore, Fig. 1 shows that both the time frame taken into account and the Degree of Semantics (DoS) involved in the recognition and classification process grow as we reach a higher level of the pyramid.

At the motion level, tasks such as movement detection, and background extraction and segmentation are faced [41, 72, 89]. Using a time frame in units of frames, a lot of research is done in the field of gaze and head-pose estimation [53, 84, 91, 92, 99, 105].

At the action level, human motion is not only detected, but also recognised in order to establish what a person is doing or with which objects the person is interacting. In a time frame in units of seconds, simple human activities; like sitting, standing or walking [8, 24, 57, 54, 133]; can be recognised; as well as location changes in indoor and outdoor environments [74].

At the activity level, a set of multiple actions is classified in order to understand human behaviour in a time frame from tens of seconds to units of minutes. ADLs are recognised; like cooking, taking a shower or making the bed; as those require tracking and classification of a sequence of actions in a particular order. This way, the sets of actions are understood as activities, where these activities are either the goals or the results of their involving 
Table 1: Classification of tasks according to the degree of semantics (DoS) involved

\begin{tabular}{|c|c|c|}
\hline DoS & Time lapse & Description \\
\hline Motion & frames, seconds & $\begin{array}{l}\text { Movement detection, Background subtraction and } \\
\text { Segmentation; Gaze and Head-pose estimation. }\end{array}$ \\
\hline Action & seconds, minutes & $\begin{array}{l}\text { Establish with which objects the person is interact- } \\
\text { ing. Recognise simple human primitives (sitting, } \\
\text { standing, walking, etc.) }\end{array}$ \\
\hline Activity & minutes, hours & $\begin{array}{l}\text { Tasks that consist of a sequence of actions in a par- } \\
\text { ticular order. ADLs are recognised (e.g. cooking, } \\
\text { taking a shower or making the bed). }\end{array}$ \\
\hline Behaviour & hours, days, ... & $\begin{array}{l}\text { Highly-semantic comprehension comes into play } \\
\text { (ways of living, personal habits, routines of ADLs) }\end{array}$ \\
\hline
\end{tabular}

human actions.

At the behaviour level, highly-semantic comprehension comes into play. Within a time frame ranging from days to weeks; ways of living, personal habits, and timetables and routines of ADLs can be analysed. At this point, abnormal behaviours and anomalies can be detected, for instance, in order to be able to detect senile dementia prematurely [48, 67, 68].

Table 1 summarises the different Degrees of Semantics considered by the taxonomy, along with some examples. Not only time frame and semantic degree grow at higher levels of this hierarchy, but also complexity and computational cost lead to heavy and slow recognition systems, as each level requires most of the previous level tasks to be done too. For this reason, level abstraction is key in order to analyse only the necessary parts and avoid redundant processes. Human tracking is the best example because it can be approached at least at the first three levels, having different tracking targets and using different kinds of features from the underlying levels. Therefore, tracking will not be discussed in this paper on its own, but tracking approaches from the analysed works will be mentioned when significant.

\section{Pose, gaze and motion estimation}

Motion recognition is the basis for estimation of human pose and gaze direction (also referred to as focus of attention) and for further HBA tasks. Motion can be seen as a series of poses along the time; the human body is an articulated system of rigid segments connected by joints (as models used 
in $[5,101]$ assume); and human motion is often considered as a continuous evolution of the spatial configuration of the segments or body posture (as stated in [55] and exploited in [5, 101]). On the other hand, the gaze can either be seen as a line in the 3D space or a cone; or, if working only in the horizontal plane (as some works do, as seen later on); a direction and an angle.

\subsection{Pose estimation}

There are handfuls of previous surveys which analyse and describe "human motion" or "human behaviour understanding" [41, 88, 43, 87, 121]; earlier works, as is logic, review lower level techniques (e.g. the work by Gavrila [34]); and later works review also further abstraction levels, approaching more to what is understood as behaviour by the taxonomy employed in this review.

Gavrila's work deals with a great amount of techniques which are aimed at providing the area of interest, without an explicit shape model by using either segmentation (as background subtraction or skin detection) or Haar wavelets (and PCA).

When shape models are used, XYT volumes are built (which reveal characteristic patterns, see Fig. 2) [35]; stick figure models are also employed. Others use a 'blob finder': each blob is defined as the shirt, pants, hands and head of a person, and these are found in an image. There is also a subsection dedicated to 3D body modeling.

When it comes to action recognition, the document presents a variety of techniques, which can detect actions (at the level defined in the taxonomy employed throughout this document). Most techniques are either based on Dynamic Time Warping (DTW), as well as Hidden Markov Models (HMM).

In the work by Moeslund [71], bigger emphasis is given to the recognition of motion and actions, whereas activities and behaviours are treated to a minor extent. In a later work by the same author [72], activity and behaviour recognition are dealt more widely (this work will be mentioned with more detail in further sections).

Nevertheless, the techniques revealed in [71] allow a greater understanding of current methods, and thus, both this and [34] can be used to introduce the early phases of behaviour analysis which is widely seen as a post processing, or a step to follow after prior segmentation and low-level analysis.

The work by Moeslund et al. [72], from 2006, can be divided into two major parts: pose and motion capture, and action recognition. In the first 


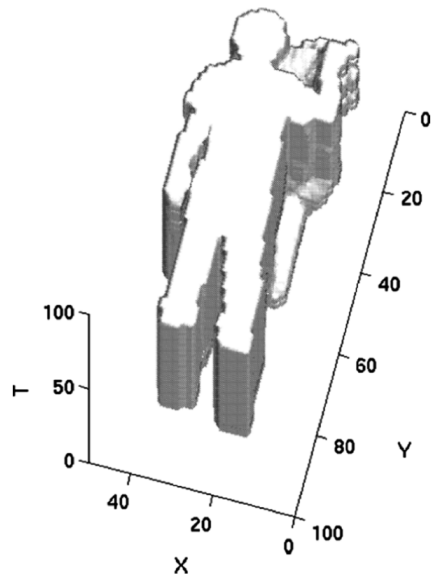

Figure 2: Gorelick et al. [35] XYT volume (reprinted from [118]).

part, a series of works are introduced and three different phases namely model initialisation, tracking and pose estimation are presented. These three phases are then subdivided into families of approaches due to some similarity; or they are divided into subphases.

Model initialisation, which captures prior knowledge of a specific person in order to constrain tracking and pose estimation, is thus presented from different points of view: the kinematic structure being used (e.g. a skeleton with a number of joints with specified Degrees of Freedom - DoFs-) and how it is initialised; the technique used to approximate the subject's shape (either using simple shape primitives such as cylinders, cones, etc., or a polygonal mesh); or appearance (presence of skin, clothing, the use of body part detectors for different types of limbs or the trunk - see Fig. 3-; similarly to what is described in $[5,14,31,33,86,101,106])$.

Figure-ground segmentation techniques (tracking) are then introduced according to the approach being used. Six different families are seen: 1) Background subtraction; 2) Motion-based segmentation; 3) Appearance-based segmentation; 4) Shape-based segmentation; 5) Depth-based segmentation; and 6) Temporal correspondences.

Pose estimation techniques are introduced next, and they are classified into three groups as in [71], according to the presence and use of an explicit model: model-free, indirect model use, direct model use.

In what is related to model-free approaches, research about 'body plans' and combinations of body part detectors are presented [128] (more modern 


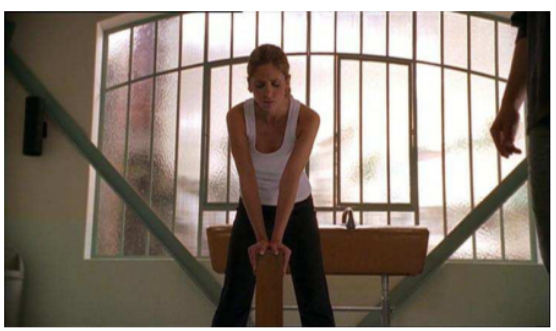

a

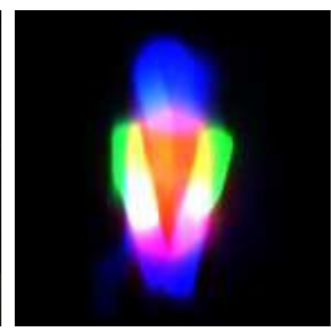

b

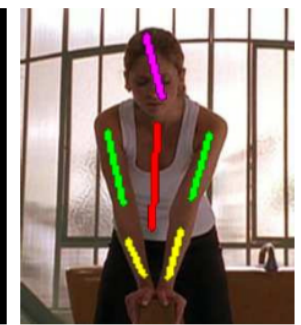

c

Figure 3: Upper-body part detector results as stated in Ferrari et al.'s work (reprinted from [33]).

works include $[5,14,31,33,101])$. Furthermore, techniques that are examplebased are presented too; these use either a representation of the mapping from $2 \mathrm{D}$ silhouette sequences in image space to skeletal motion in 3D pose space [98] or direct lookup of silhouette sequences for recognition $[1,40,104,108]$. There are two main drawbacks to this family of techniques: 1) when using 2D silhouettes as key poses, a constraint on the point of view is added, which limits recognition to that exact point of view; to overcome this, different viewpoints can be added into the database, which can be cumbersome and lead to worse inter-class recognition; and 2) the larger the number of classes, the worse the recognition will work (see for instance [124]).

In indirect model use techniques, in turn, methods that use direct reconstruction of both model shape and motion from the visual-hull are revealed $[23,70]$.

Finally, direct model use techniques are seen; under this set of methods, multiple view 3D pose estimation using gradient descent techniques, and more recently particle filtering (a state space search reduction is used), are of interest. An evolution of the works presented under this section of Moeslund's work, can be seen in the papers by Bandouch, Beetz, et al. [7, 9]; which are commented later on. To end with direct model use techniques, monocular 3D pose estimation and learnt motion model methods are presented.

A more recent survey is the one by $\mathrm{Hu}$ et al. [41]; this paper is highly comprehensive, as it starts with motion detection and object classification and tracking, but also deals with HBU; it divides the approaches to this task depending on the techniques used (Dynamic Time Warping, Finite State Machines, Hidden Markov Models, Time-Delay Neural Networks, Syntactic Techniques, Non-deterministic Finite Automata, Self-Organising Maps). 
Then, it also reveals techniques aimed at describing the behaviours using natural language. After that, it goes on to personal identification by means of different methods including all sorts of biometrics (limb lengths, speeds, gait, height, weight, face recognition, etc.)

It also takes the fusion of data from multiple cameras into account, and explores future developments. In this section, however, only the first phases, which deal with motion detection, are of interest. Here, pose estimation algorithms which prove useful for the tasks described in Sec. 1, as well as Human-Computer Interaction (HCI), will be revealed.

In the literature, very different approaches $[64,117]$ for foreground/background segmentation can be found, which are very popular in computer vision tasks. Such techniques are aimed at determining the position of the moving objects in a scene. Frame differencing might be the earliest of these techniques, followed by other background subtraction techniques, based on a wide variety of models. Statistical models could be dealt here, such as Adaptive Background Mixture Models (using mixture of Gaussians) [110], Wallflower [117], or others [64, 117].

Segmentation algorithms, if not accompanied by further techniques, provide only very basic pose estimation (as a silhouette or area), which can be only used as information on where the subject is. Depending on the task to be done, this could be enough; otherwise, further estimation refinement techniques are at hand. For example, Lv \& Nevatia [60] take a single silhouette from a video, and with it, they are able to recognise actions from a set of previously learnt examples.

Other ways to determine the position of objects in a scene are based on object detection, without segmentation. In the work by Dalal \& Triggs [25], descriptors are generated for image windows. These provide a means for recognition of certain shapes (the human body). These descriptors are then fed to a classifier for training. After that, in the test stage, the classifier determines whether each sample in a window is of the object class. Applying this method, object presence in a scene can be confirmed. It can also be applied to determine the kind of object present in the bounding box obtained from a previously applied segmentation algorithm.

Segmentation-free pose estimation techniques are based on human models (or object models in general); either complex anthropomorphic 2D or 3D models (i.e. [66], used in [7]), or approximations to it (cylinders or ovals [66], skeletons and stick figures [19], etc.). The main difficulty that arises when using no segmentation, is how to find a way to determine where the joints of 
people are [114].

Nevertheless, those models can also be used with segmentation; with that coarse knowledge extracted from the previous phase where silhouettes are estimated, recent works propose to infer finer pose estimation. To do so, Boulay et al. [13] propose a system which, using a single camera, is able to determine the pose from a set of poses. Using the data from the segmentation phase, they proceed to estimate 3D cues, such as depth and others. With these, and using a virtual 3D environment with the same size and camera position, they calculate how the person might appear as a 2D silhouette depending on the pose in those exact coordinates. After that, the virtually generated poses and the actual pose are compared with a histogram comparison algorithm, which yields the most probable pose as a result. The whole workflow runs in real time (4 to 15 fps). Further work [134], applies the described technique in an AAL scenario. This way, there are works which choose to cover the blob with an elliptical model in order to extract conclusions based on the direction of the major and minor axis of the ellipse and the length ratio between them (see [74]). For instance, this method enables to detect if the individual is standing, sitting or lying. As a result, the system is able to detect possible falls.

A 3D-volume approach is presented by Anderson et al. [4], which uses $2 \mathrm{D}$ silhouettes extracted from calibrated cameras to mount what is called a 'person voxel'. They further apply fuzzy logic to classify three different body poses (upright, in-between and on-the-ground); and thus be able to detect falls.

More recently, other works based on similar approaches have appeared $[7,9]$; these allow a very detailed pose estimation. These are based on the use of three cameras, instead of one, without prior knowledge of the room model (no calibration), the only requisite being that the cameras are set so that they see the object from different perspectives (ideally orthogonally, with non-overlapping views). With such a scheme, the MeMoMan project [7] has been able to determine very fine pose estimation in real time. The technique is similar to $[13,134]$. Using the silhouettes (in this case three), a hierarchical 3D human model is applied, which fits into the observed silhouettes. To reduce the dimensionality, mathematical models are used, which allow faster pose estimation. Furthermore, the system has been applied in a kitchen scenario, for ADL analysis [9]. 


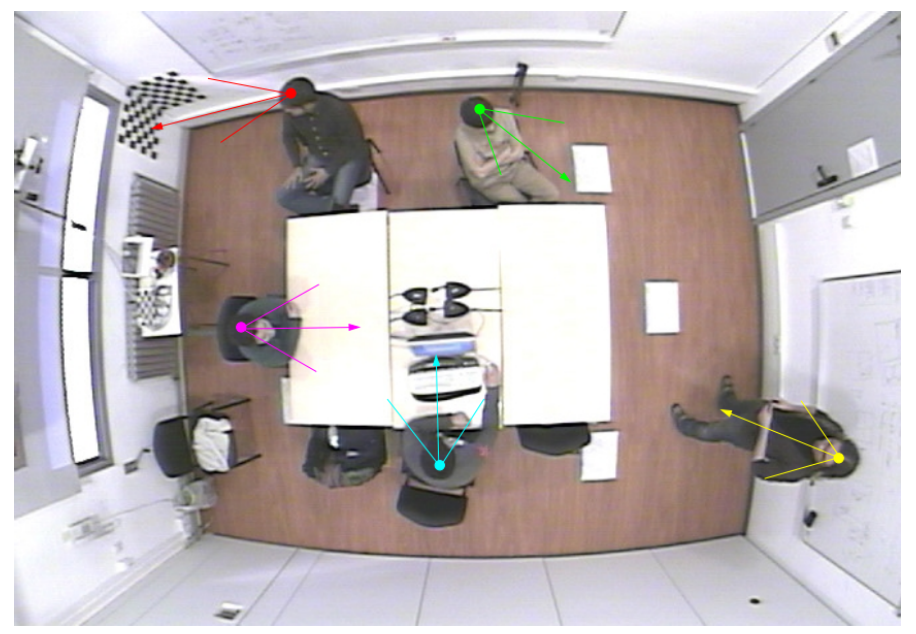

Figure 4: Focus of attention estimation of a group of people (reprinted from [17]).

\subsection{Focus of Attention/Gaze estimation}

So far, only works related to body pose estimation have been discussed. In the field of AAL, other body cues are interesting too, such as gaze direction (or focus of attention), which provides further information related to what is being done in the scene [62]. Gaze estimation can also be used to detect distraction, or abnormal situations. On the other hand, different researchers treat gaze differently, it can be understood as a line in the $3 \mathrm{D}$ space (a beam-like approach) or a cone [17]; or, if working only in the horizontal plane $[53,84]$, a direction and an angle (compass-like approach). Some of the reviewed works use gaze estimation for different purposes. Canton-Ferrer et al. [17], for instance, use gaze estimation for attention analysis in smart classrooms or offices (see Fig. 4). Doshi \& Trivedi [28, 29] use gaze estimation to detect driving styles and distractions.

In [62], gaze estimation is seen as an additional cue for video annotation and understanding. The authors present various techniques with different degrees of fineness; by either using simple information about left or right head orientation, or yaw and pitch angles along with inferred depth ( $\mathrm{z}$-axis) information. For the upper-body and head detectors described, Felzenszwalb et al. model [32] is used.

Head pose estimation can be understood as an alternative term for the detection of the focus of attention of a specific person, as the gaze of a person is determined to some extent by the pose of his/her head [62]. In Launila \& 
Sullivan [53], different colour and shape properties are combined in order to estimate the pose of the players' heads in a soccer match. This work is part of a greater project whose final objective is to reconstruct a whole soccer match in 3D and in real-time. This would make it possible to watch the match from any point of view and solve conflictive situations at refereeing.

Within the field of head pose estimation, in Ozturk et al. [84], gaze direction is obtained in steps of 22.5 degrees in wide indoor areas such as airports and malls. They develop the first solution to the head pose estimation problem using only the data proceeding of a single $2 \mathrm{D}$ camera. A two level particle filter made up of colour and edge histograms is used for tracking. Afterwards the individual silhouette is matched to one of 16 patterns by using shape descriptors and SIFT points.

\section{Action recognition}

After the initial step of motion detection, and pose or gaze estimation; basic actions can be understood as a series of motions detected; either in the whole or in some parts of the subject's body (arms, legs, head, etc.); or in some areas of a room (living room, kitchen, bathroom, outdoors, etc.). Such actions can be recognised because of the different body poses which are involved, and the variation through short periods of time.

In order to understand the difference between an action and an activity, not only time lapse is taken into account; the objects and people involved are important too. For instance, a person manipulating an object is performing an action (say, opening a lid); while several of such actions, performed with different objects, compose what is called an activity (e.g. cooking a meal).

This section is centered in the former, and as such, it will describe the works and techniques which are either based on or aimed at recognising $a c$ tions. Various categorisations have been found for action recognition methods $[55,118,122]$. These works classify action recognition based on the approaches used for action modelling.

According to Turaga et al. [118], approaches for modelling actions can be categorised into three major classes: nonparametric, volumetric, and parametric time-series approaches (see Fig. 5).

Inside 2D-template-based approaches described in [118], temporal templates are estimated for each action class. The work by Bobick \& Davis [12] presents two mechanisms called Motion-Energy Image (MEI) and MotionHistory Image (MHI), respectively. The MEI is a binary image which rep- 


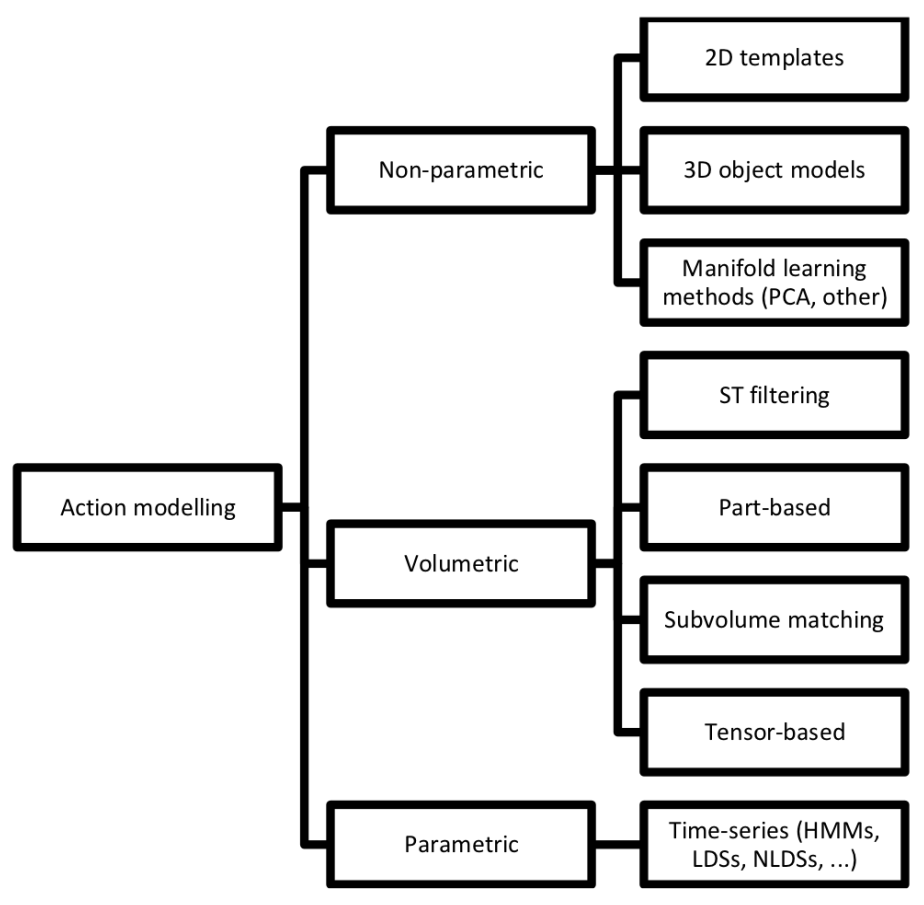

Figure 5: Turaga et al.'s [118] action modelling classification.

resents where motion has occurred, while the MHI is a scalar-valued image where intensity of the pixels is a function of the recentness of motion (Fig. 6).

Also under this class, Ben-Arie et al. [11] reveal a technique, in which data representing the angle of the limbs is extracted from the silhouettes. Only a few representative poses are taken into account for the learning process and stored in a database. Indexing is performed, so that searches relating only one specific limb can be performed over the action model database. For recognition, after extracting each limb's pose, a voting scheme is used, which returns the most likely action.

In volumetric approaches, in contrast, features are not extracted on a frame-by-frame basis, but instead the whole video is considered as a 3D volume of pixel intensities, and standard image features are extended to deal with the 3D case [118]. For instance, Laptev [51] proposed an spatiotemporal (ST) generalisation of the Harris interest point detector, to model and recognise actions in space-time. Niebles et al. [78] use such interest points in a bag-of-words model in order to represents actions. Clustering of the features and classifiers (such as SVMs, graphical models, etc.) can be 


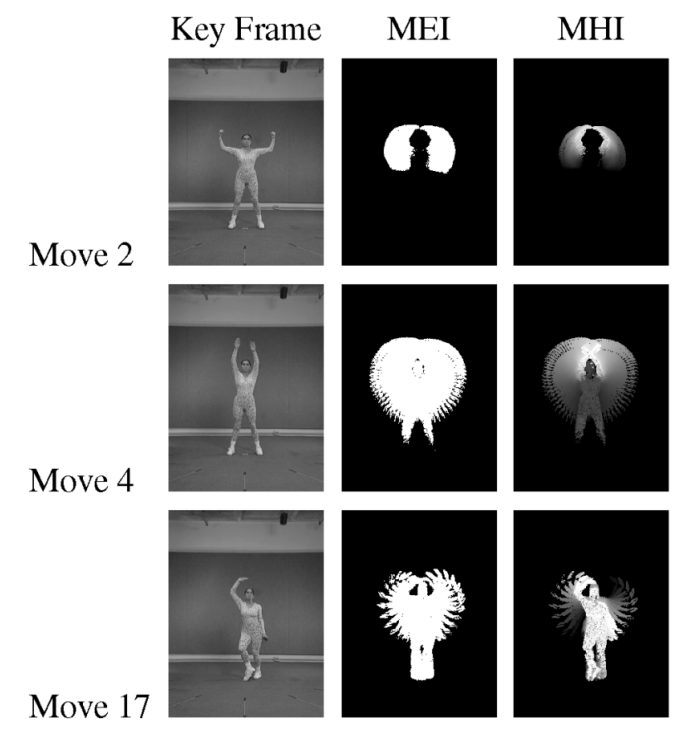

Figure 6: Examples of MEI and MHI images (reprinted from [12]).

applied afterwards. Based on similar ideas, Ryoo \& Aggarwal [100], propose a kernel function to measure similarity between pairs of videos.

Similarly to [118], Weinland et al. [122] categorise the works in action recognition depending on the way they model the actions being recognised. The two approaches mentioned are 'model-based' or 'template-based' ('holistic'). According to the authors, the former approach assumes a known parametric model, typically a kinematic model, and actions are represented in a joint or parameter space. This approach shows difficulties to estimate the pose correctly without the use of markers or other means of easing the task.

In contrast to this, in the later approach, the use of spatio-temporal shapes as action templates reduces the burden. Actions are then modeled using information retrieved from the images (such as silhouettes, optical flow, etc.). For recognition, comparison between the observation-inferred templates and learned templates is required. The authors point out the limitation of this later technique: both learning and recognition need to be done under similar camera configurations.

In [55], a similar categorisation is made. Here, the approaches are either based on the extraction of action descriptors from the silhouette sequences (similarly to 'model-based' approaches seen in [122] or the non-parametric 
methods shown in [118]); or based on the extraction of features from each silhouette and model the dynamics of the action explicitly (again, similarly to the aforementioned 'holistic' approaches).

The approach proposed by the authors in [122] takes advantage of the template based methods, but without this last restriction, thus they avoid the need for view-specific training databases. Action recognition is performed from 2D cues, while in the learning stage, the HMM states are not represented by a single 3D exemplar; this accounts for different body proportions, style, or clothing. In [122, Sec.2], a state of the art relating view-independent action recognition can also be found.

Another application of action recognition is that of smart surveillance systems. Cheng et al. [21], for instance, propose a system which is used both for parking lot surveillance and indoor tracking in which primitive actions are logged. The technique they present is based on Histogram Oriented Occurrences (HO2), which is described as “(..) a new feature that captures the interactions of all entities of interest in terms of configurations over space and time. HO2 features encapsulate entity tracks, inter-object relationships and the context of the environment into a spatial distribution that characterizes the corresponding event." These new features allow easier multi-agent event recognition; in contrast to simpler feature vectors which yield HMMs with too many nodes. The proposed feature is based on the ideas of Histograms of Oriented Gradients (HOGs), presented in $[25,58]$ and seen in the previous section; and the Shape Context descriptor, described in [10]. After the HO2 features are calculated, they are then fed to an SVM classifier, in order to detect the different event types (arrivals, departures, trunk loading or unloading, etc.).

In [22] Cherla et al. use DTW along with a two-component feature vector, whose elements are: the width profile, calculated from the silhouette's bounding box; and some spatiotemporal features, such as the displacement of the centroid (in X and Y), and the standard deviation (also in both axes). Because of the dimensionality reduction applied, actions performed sidewise (e.g. walking) can be detected better than actions which take place in a frontal manner (except for hand waving and other actions which involve sidewise movements of the limbs).

Three works by Oikonomopoulos et al. [80, 81, 82] describe techniques aimed at recognising basic actions. The earlier work [81] reveals a technique for aerobic exercises action recognition by means of a sparse representation based on spatio-temporal salient points; these are obtained using a method 
presented by Kadir \& Brady [47] that takes scale variance into account, among other considerations. A distance measure between two points is also introduced, which is based upon the chamfer distance.

In [80], the authors deal with the use of $B$-splines as a means of describing the movement of points along the time axis; these descriptors will feed a codebook afterwards, which allows further recognition to be performed. The later paper [82], is based on ST shape model. The goal is to detect actions without prior segmentation. In the paper, a ST segmentation is performed, and a voting scheme is used. The technique is robust against partial occlusions, and multiple activities can be detected if performed simultaneously.

Within the Prometheus (FP7) project, a work by Quintas et al. [90] describes the use of Concurrent Hidden Markov Models (CHMMs), for the detection of ADLs in a smart home environment. Although the work talks about behaviours, the events described along the paper correspond to what is classified as actions by our taxonomy. Thus, this research falls into the category treated in the current section.

\subsection{Data Fusion}

Other works base their motion recognition techniques in other kinds of sensing, which are more intrusive than the methods described up to this point; these are based either on other kinds of vision, or on other kinds of sensing.

One remarkable example is 'wearable vision', which is a semi-intrusive scheme, in which a camera is mounted on the person's shoulder, or attached to the frame of their glasses, etc. Papers reviewed using such techniques $[93,111,112]$ emphasize how wearable cameras avoid the body to occlude what is being managed with the hands (see Fig. 7). They also point it as a more natural approach because activities are performed looking at what is being done. These head-mounted cameras, allow the researchers to work with 'first-person' images, in which they see the hands of the user, and the object interaction along their ADLs. The only drawback of such a scheme is that hands provoke occlusions too, just as the body does in non-wearable cameras. Moreover, cameras of such kind need to be improved, as they can be really cumbersome for the final users.

Other sensing devices are also used in diverse works $[2,8,63,109,132]$, or a combination of vision and RFID tags [129] either as a direct way to recognise the objects being manipulated, or as a means for supervised learning of visual object appearance (using the correspondence between the RFID and 


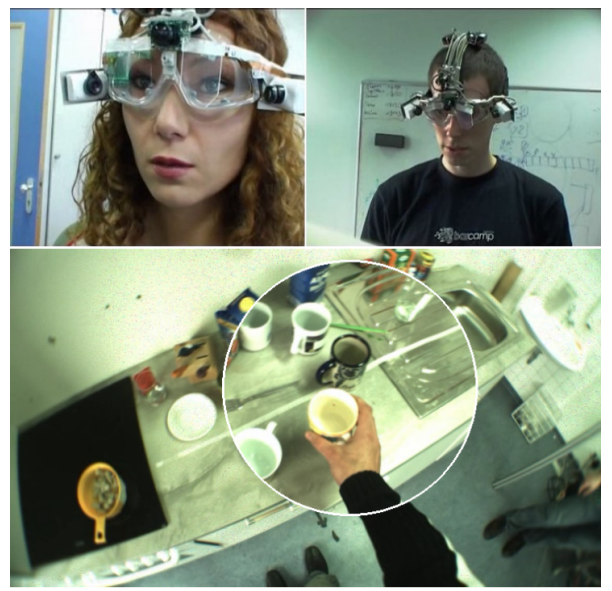

Figure 7: A gaze directed camera used in [111]; the camera itself is shown in the upper images. The lower image shows a superimposed camera view generated by the device (reprinted).

visual data). Yang et al. [132] use a sensor network in order to determine the position of the body by means of a network of wireless motion sensors. In [109], data from the wearable camera is enriched with data from the Inertial Measurement Units (IMUs), which are worn by the subjects in a form of bracelets. Furthermore, Maurer et al. [63] recognise activities by using only one bracelet (called $e$ Watch) which they attach at different body parts for comparison. In [8] Bao \& Intille use bi-axial accelerometers attached to different body parts in order to determine the wearer's ADLs (mostly actions, but some complex activities too). Altun \& Barshan [2] also use Inertial/Magnetic Sensor Units (IMSUs) in order to determine the actions being performed. This last work also emphasizes the fact that vision and IMSUs are not exclusive. It also mentions some other papers in which vision and the mentioned sensors are used in hybrid systems [114], or as a method to check the correct classification when using only data from IMSUs, as in [3, 75, 97]. Kwapisz et al. [49] propose the use of tri-axial accelerometer-equipped smart phones for the same purposes.

Li et al. [55] take the use of other visual sensors into account. Their technique, based on 3D point clouds, deals with the recognition of actions by means of bags of three-dimensional points, obtained at 15 fps by a depth camera that acquires the depth through structured infra-red light. The proposed method obtains a highly reduced subset of the point cloud, based on 
the observation that pixels in the silhouette boundary (contours) are the most relevant ones, as they carry more information on the observed body's shape. Action recognition is then performed by means of action graphs, which are described in a previous work by the same authors [56].

\section{At Activity Level: Activities of Daily Living}

At this level, the goal of the recognition is to classify a sequence of actions into their targeting activity. For instance, some children could be moving their legs and their arms, jump and run, and interact with a ball. But, what are they playing? At this moment, semantics come into play and understanding the compound of actions is what gives actual value to the recognition. How can we distinguish a basketball game from a volleyball game? This is the reason why the particular order of the actions and the interacting objects are key for activity recognition. If we are able to distinguish the type of ball, the net from the hoop, or even that in basketball, the player runs while bouncing the ball by tracking the involved actions; we are taking into account a larger time frame and a significant higher degree of semantics than in previous HBA levels.

In particular, recognizing ADLs in smart homes can lead to understand what a person is doing, and enables monitoring of completeness and correctness of these activities. In this matter, Mihailidis et al. [68] are able to track the activity of hand washing to assist older adults with dementia. Multiple orders in the process can be correct, but not of all of them; their system is able to prompt the user if a necessary step is missing or the order of the implied actions is unacceptable. Vision is used as the only sensor in the developed system for two purposes: 1) tracking of hand location; and 2) tracking of step-specific object locations. In previous works [69], hand washing was tracked with switches and motion sensors. This way, the system could infer whether the hands were in the sink or if soap was used. Nevertheless, the authors explain that although this data was reliable, too little was known about the user and the environment. Even if taps are on and the motion sensor indicates that the hands are in the sink, there is no guarantee that the individual is actually washing his/her hands.

Related to this type of activity recognition, Wu et al. [129] stand out in activity recognition based on object use. As mentioned before, these authors define activities as combinations of actions and objects and intend to recognise and track object use in order to infer human activities. Object models 
are acquired automatically from video, whereas object identification is based on RFID labels. At the learning phase the user wears a RFID bracelet which reads the RFID tags attached to the surrounding objects in a home environment. Assuming that the object being moved is always the object in use and that only one object is being moved at a time, the system learns the relationship between the segmented image and the active RFID tag using a dynamic Bayesian network. As arms and hands move with the objects, skin filtering is applied beforehand. At the test phase, the system works without the RFID data as objects are recognised by detecting SIFT features within the segmented area. These key points are matched based on maximum likelihood to the previously trained SIFT points. As the number of possible SIFT features is very high; clusterization is applied, using the $\mathrm{K}$-means algorithm, in order to obtain a delimited histogram of SIFT features for each object.

In [133], activity recognition is approached differently. The individual silhouette is obtained at different positions of a living room. Grouped into 10-20 prototypes each silhouette stores its centre, width and height and is manually labeled with a location. A fuzzy inference method is used to estimate the most likely physical location of test silhouettes. Location estimation and previously assigned coordinates enable average speed measurement, which is used besides location in order to recognise human indoor activities. A Hierarchical Action Decision Tree (HADT) is used to classify human actions using multiple levels. At the first level, human actions are classified based on location and speed. With $\mathrm{K}$-means, clustering feature patterns are obtained; and activities of daily living, like walking or visiting the bathroom, are recognised; this is achieved by using the $\mathrm{K}$-Nearest Neighbour (KNN) method. At the second level, a more precise recognition of activities like washing, eating or cooking is achieved. From the individual silhouette, the smoothed boundary of the human body is extracted using a snake model, which is represented with $\mathrm{Hu}$ Moment Invariants (HMI) [130]. This way, the temporal variation of the HMI values are used to measure the level of body motion and instead of tracking single actions, activities are inferred based on how active the person is. The third and last level is only used when a person remains at the same physical location while he/she is moving constantly; this happens, for instance, at exercising. In this condition, further recognition is needed, and primitive visual features are used. By partitioning the video frames in blocks of $8 \times 8$ pixels, motion is analysed individually at each block. This way, for a $640 \times 480$ pixels video frame, 4800 points are taken into account to characterise low-level motion. Locally Linear Embedding (LLE) 
is applied to reduce the dimensionality of these feature vectors into a small set of composite features. These features are handled as a trajectory and matched using distance correlation and KNN classification.

When dealing with different types of sensors in smart homes, uncertainty of sensor data needs to be considered. In [38], belief in sensor data is deeply analysed. When dealing with binary sensors; like movement detectors, contact switch sensors and pressure mats; the sensor data could be erroneous due to a variety of reasons. The sensor itself could be faulty; the data could be approximate, as the exact value is impossible to be measured because of the very nature of what is being measured; or the system could have corrupted the data while reading and sending it to the upper level. Dempster-Shafer's theory of evidence $[27,103]$ is considered to be able to represent ignorance due to lack of information, and to aggregate belief when new evidence is obtained. Kitchen door sensors and motions sensors are used to recognise ADLs, like making a drink (differentiating between hot and cold) or making breakfast (cereals, toast or eggs). With multivalued mapping, rules are built in order to know which elements are involved in which actions. For instance, making a tea implies a tea bag necessarily, but milk can be optional. This way, evidence is assigned to these rules, and it is possible to infer the most likely activity given the certainty of the current sensor data.

Nicolini et al. [77] collected data from a couple who lived at a custom built condominium for a period of 10 weeks. Several hundreds of sensors, including audio-visual recording, collected data in order to be analysed in intervals of 30 s with 15 s of overlapping between each consecutive interval. ADLs like watching TV, grooming, reading and using the phone are recognised with multi-labelled prediction. Besides sensor data, average activity duration is used to train SVM classifiers, one for each activity. As a refinement stage, Conditional Random Fields (CRF) [113] are applied to model sequential observations and recognise completed activities among combinations of local on-going activities. Validation was done using leave-one-day-out cross validation and area under the ROC curve (AUC) as a figure of merit reaching a result from 81 to $97 \%$.

So far, we have presented vision-based activity recognition systems which use global features, like image foregrounds or individual silhouettes; or local features, i.e. keypoints, as salient points or corners. The field of Image Analysis and Processing provides a wide range of image features and types of key points; these are used in diverse application areas and present different advantages and drawbacks [46, 119]. Although most popular key points 

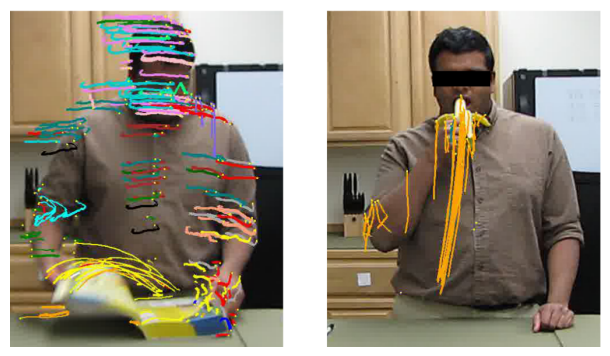

Figure 8: Labelled example flows from Lookup in Phonebook and Eat Banana (reprinted from [65]).

techniques as SIFT and SURF are applied frequently at activity recognition, concrete keypoint-based features for this purpose are available too. Velocity history of tracked key points is used in [65]. Interest points are chosen based on gradient difference and tracked using a KLT tracker [59]. This way, about 500 features are tracked at a time, replacing missed points on the fly; their velocity history is used as the basic feature. Classification is based on a generative mixture model and experimentation data is presented on the KTH dataset (see Sec. 7.2), as well as on an own dataset where activities like writing a phone number on a whiteboard or peeling a banana are recognised (shown in Fig. 8).

In [54], Lester et al. proposed a personal activity recognition system for health-care purposes which satisfies three key restrictions: 1) data is collected from a single body sensor and it is not required to be from the same point for every user, 2) personalization could enhance results but should not be required, and 3) should be effective even with a cost-sensitive subset of the used sensors. With these pre-requisites, data of 10 male and 2 female individuals were collected in order to recognise 8 different physical activities; like sitting, standing, walking (what we classify as actions); but also activities with higher semantics and interactions with objects are recognised, as for instance, brushing teeth or riding an elevator up or down. Volunteers wore a multi-modal sensor board at wrist, waist or shoulder. This way, data from following sensors were collected simultaneously: microphone, light phototransistor, 3-axis digital accelerometer, 2-axis digital compass, digital barometer/thermometer, digital ambient light (IR and visible) and digital humidity/temperature. Out of these data, 18000 samples per second are reduced to 651 features; these include linear and log-scale FFT frequency coefficients, cepstral coefficients, spectral entropy, band-pass filter coefficients, 
correlations, integrals, means and variances. Their recognition algorithm is hybrid in the sense that models of the underlying distributions of the data classes and class boundaries for discriminative techniques are learned. At a first level, a custom boosting is applied to select the best features iteratively and to feed an ensemble of discriminative static classifiers with the most discriminative sub-set of features of each activity. At a second level, HMMs are used to recognise activities based on the class probabilities obtained from the static classifiers.

Activities of interest to medical professionals; such as toileting, bathing and grooming; are recognised in [115]. Tapia et al. introduce an interesting approach for labelling train samples manually: the Context-aware Experience Sampling Tool. Subjects carry a PDA that is used as a timing device to trigger self-reported diary entries. The PDA asks the user for information when a certain number of changing sensor values are detected; multiple choice questions can then be answered by the user. This way, the system is told what activity he/she is performing right now, and for how long he/she has been doing this activity. Tests have been performed for 14 days in two one-bedroom apartments with one subject each. 77 state-change sensors collected data at doors, windows, cabinets, microwave ovens, refrigerators, toilets, showers, water taps, etc. In this work, naïve Bayesian classifiers are extended to incorporate temporal relationships among sensor firings and implemented in two versions: The first is a multi-class naïve classifier, in which the child nodes either have exist or before attributes. This means that temporal order is being considered: if a particular sensor should fire when performing a certain activity (exists), or if it should fire before another particular sensor. The second version consists of multiple binary naïve Bayes classifiers, one for each activity. In contrast to the first version, this one is not mutually exclusive. Finally, activity duration is considered too, applying feature windows individually for each activity.

In order to describe ADLs consistently at several abstraction levels, Beetz et al. [9] proposed so called Automated Probabilistic models of Everyday Activities (AM-EvAs). AM-EvAs consist of automated activity observation systems, interpretation and abstraction mechanisms for behaviour and activity data, as well as reasoning and query systems that enable AM-EvAs to answer semantic questions about the activities. This way, these models have information about the involved actions and sub-actions, objects, agents who performed the activities, location and time. The purpose of these models is to build a knowledge-based framework to combine observations of human 
activities with a-priori knowledge about actions, and to make the classification and assessment of actions and situations objective. Therefore, action patterns of different activities are learned from several subjects in order to support singularities. The activities are observed with vision-based fullbody motion tracking, RFID tags and magnetic sensors. The sensor data stream is segmented and classified with action classifiers which recognise movement primitives. This data is combined with time intervals and events, and represented in a first-order logic language. Probability distributions are represented either with Bayesian — or with Markov - Logic Networks. In

conclusion, AM-EvAs make it possible to train objective knowledge-based models to save meta-information from activities and query following types of questions: 1) Relational knowledge, like Which is the whole pose sequence of a table setting activity?,2) action related concepts, like Where is the table setting activity performed?, and 3) probabilistic knowledge, like Having observed that a bowl has been taken and that an egg has been cracked, how likely is it that brownies are being baked?

\section{Human Behaviour Understanding}

According to the taxonomy being employed, behaviour is understood as the highest level of complexity and time span. It is seen as a long lasting series of activities that tend to occur in a certain order. It could be seen as the observed person's daily routines. Deviations from the pattern can be seen as extraordinary, and as such, they give information about the person's evolution (say, health status or independence in the case of elderly people living alone [73]).

Under this definition, a number of works using vision as a source for activity detection are found. Although, many of the reviewed works in the field of behaviour analysis and AAL $[18,36,37,44,61,73,85,120,127]$ are based on other sensor devices (lights and use of appliances [18, 44, 73]; pressure mats [18]; basic motion detectors - such as door sensors or similar$[37,85,127]$; infrared sensors —SMDs - [18, 36, 44]; health monitoring [127]; etcetera).

Behaviour of people in the scene is seen in some of these works as the circadian activity rhythm (CAR); that is, the evolution of ADLs throughout the day $[120,127]$. By learning the CAR of a person, either on a weekly or a 5-day basis, the system can recognise abnormalities in the behaviour as deviations from the previously observed routines. 
From the works that do indeed include vision sources, context-aware systems $[16,24]$ and video annotation systems $[94,95,131]$ can be found. These kinds of systems can be classified under this section dedicated to 'behaviour understanding', as the main point of these is not limited to determine the activities people are performing in each moment, but to extract and infer further information from the recognised activities.

The work by Brdiczka et al. [16] deals with the recognition of situations (context). In the cited work, the authors present a system which tracks persons in 3D using cameras, and is able to extract information about the entities' pose (role); speed; and interaction with other entities (either these are people, furniture or appliances), according to the distance to them. In this arrangement, people wear headsets, which detect whether they are talking; and microphones are arranged so that ambient noise can be detected. Numeric codes are given to each possible permutation (single versus multiple people, with or without ambient noise, with or without people talking). Using these codes, which fuse all the collected information, different kinds of 'situations' are learnt and recognised by means of left-right HMMs (these situations are individual work, introduction [of various people to each other], aperitif, siesta [of one individual], presentation or [board] game [among multiple people]).

In $[94,95]$ Robertson et al. present a video annotation technique which is able to extract the commentary of a tennis sequence. To do this; position, velocity, and action descriptions are fused and fed into an spatiotemporal action recogniser, which is in turn fed to an HMM which applies a smoothing process to the output using model-based scene knowledge (which are modelled manually by using knowledge of the rules of the game and spatio-temporal constraints in the movements of players). Their uppermost layer consists of 'behaviour HMMs', which take the output of the smoothing HMM to recognise sets of activities which form a more general behaviour (e.g. baseline-rally or serve-and-volley play types in tennis). As further work, application to abnormality detection is presented.

Chung \& Liu [24] go further and propose a system which takes low-level information (such as poses, which the authors call 'activities'), and combine it with other two contexts: spatial (where does the activity happen), and temporal (when does the activity happen and how long does it last). This way, using a Hierarchical Context Hidden Markov Model (HC-HMM), behaviours are learnt and later recognised from vision sources. Their techniques are applied to AAL in the context of a nursery house in which different 


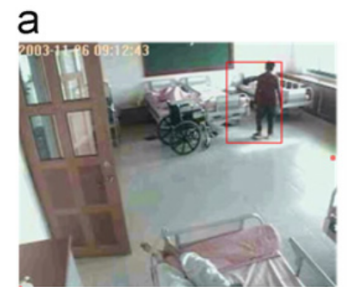

Frame 1307

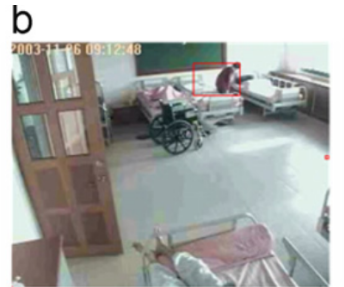

Frame 1445

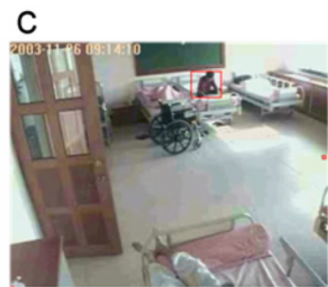

Frame 3906

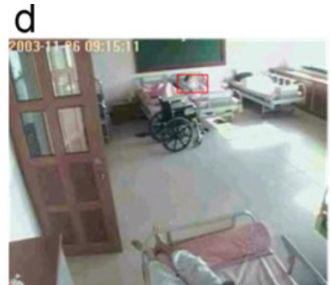

Frame 5726

Figure 9: Action and activity recognition from [24] which allows further behaviour recognition. Different actions (a, walking to bed; b, sitting on the bed; c resting on bed; d, lying on bed) imply an activity: go to sleep, which is detected by time limitation (reprinted).

behaviours are monitored (see Fig. 9). These include sequences of activities such as sit down and watch TV for a while, go to the toilet, lie on bed to sleep, eat breakfast, take a walk, etc. The normal sequences of activities are learnt from the behaviour of the monitored people, in order to detect abnormality in the expected routines (either in their duration, time of day, or location).

In [24], results are compared with the same set of videos, with other methods such as the presented in [30, 76]. Duong et al. [30], present what is called the Switching Hidden Semi-Markov Model (S-HSMM), which allows activity duration abnormality detection, although the sequence of activities is restricted both in order and in the number of activities (exactly six); the space where activities happen is also restricted, as the room is divided into a discrete number of 'cells' of 1 square meter each, and 'hotspots' are defined according to how some appliances and tools are arranged. Nguyen et al. [76] present an application of the hierarchical HMM which detects three different activities, namely have a snack, short meal or normal meal, depending on the visited spots in each action sequence.

Other works are concerned about the recognition of behaviours that include more than one person interacting in the scene. Early works in this field include [83], in which the performance of different HMM-based techniques for the recognition of interactions among two people is compared, and it is concluded that CHMMs (coupled HMMs) perform better for the task. The work introduces an interesting agent framework for the synthesis of artificial behaviours that are used for training along with real-life video data. CHMMs were introduced in [15], as a better approach for interaction modelling. Hongeng \& Nevatia [39] present a hierarchy of events along with a method for interaction modelling and recognition. Modelling is performed by means of 
action threads (associated to each actor/agent) and temporal constraints. Recognition is achieved by propagating these constraints and likelihoods in a Temporal Logic Network (TLN).

A more recent work from Liu et al. [57], extends the methods presented in [24] for the recognition of behaviours present in pairs or groups of people. By recognising the number of people present in the scene, switching between two different HMM-based approaches is performed by a switching module called 'Switch Control' (SC). Either Individual Duration HMM (IDHMM) or Interaction-Coupled Duration HMM (ICDHMM) are used as a consequence. The methods, as in the previously presented work, are applied in AAL environments (nursing homes).

\section{Useful Research Tools}

When reading a survey like this one, most readers are either initiating themselves in the field, or taking up again and looking for what advances other researchers made in the last few years. Therefore, this section is made for those who are going to start a new project and could take advantage of existing tools, models and datasets; in order to build upon, and be able to compare between different approaches. In this sense, this section will present the most used datasets, frameworks and tools in the area.

\subsection{Datasets}

When developing a new recognition systems or improving an existing one, the datasets to test need to be chosen carefully. Dataset properties vary widely, and overfitting at model training can lead to illogical results. In the field of HBA following video datasets stand out:

- HOHA - Hollywood human actions [52]: This dataset contains video sequences from 32 movies with annotations of 8 types of actions: AnswerPhone, GetOutCar, HandShake, HugPerson, Kiss, SitDown, SitUp and StandUp. Training and testing sets are provided, as well as an automatically labelled training set with approximately $60 \%$ correct labels. A second version is available with about 1200 minutes of video and four new actions in addition to the existing ones: DriveCar, Eat, Fight and Run. As video clips are taken from movies, persons in the images are focused mainly and background changes are frequent. Therefore, this dataset is very useful and challenging. Nevertheless, it 
should not be forgotten that this type of images is difficult to obtain with regular surveillance cameras.

- KTH human motion dataset [102]: This action database contains six types of human actions performed by 25 subjects at four different scenarios. Walking, jogging, running, boxing, hand waving or hand clapping are performed in over 2000 sequences. Backgrounds are homogeneous and free of clutter. Video files are classified by actions, so that unwanted actions can be excluded easily. In contrast to the HOHA dataset, background segmentation is much easier with this type of images; and annotated actions can be placed at the same semantic abstraction level.

- Weizmann human action dataset [35]: Gorelick et al. used static front-side cameras to record single human motion from 10 subjects in different environments. About $340 \mathrm{MB}$ of video sequences are available; performed actions include walking, running, bending, hand waving and different types of jumping. The corresponding background sequences, with no subjects, and the subtraction masks - either with post-aligning or without it - are available too. The system is based on space-time features and is able to recognise complex actions like ballet movements.

- INRIA Xmas motion acquisition sequences [123]: This dataset includes $390 \times 291$ pixels video images recorded from five different angles. 11 actors performed 13 actions: check watch, cross arms, scratch head, sit down, get up, turn around, walk, wave, punch, kick, point, pick up, throw over head and throw from bottom up. These actions were performed three times each, in an arbitrary chosen angle in relation to the view-point. Backgrounds and illumination settings are static and free of clutter.

- TUM kitchen dataset [116]: This dataset targets ADLs at a kitchen scenario at a low action level. Table setting is performed by several subjects in different ways; some transport items one by one; and other behave natural, grasping several objects at once. Video images have a resolution of $384 \times 288$ pixels at $25 \mathrm{fps}$; and motion capture data, extracted with a marker-less full-body tracker, is provided. Furthermore, RFID tag readings from fixed readers at the placemat, the napkin, the plate and the cup; and sensor data from magnetic sensors at doors and 
drawers are available. Each frame has been labeled manually and separately for the left hand, the right hand and the trunk of the person. Among others, actions like carrying an object, standing still, reaching, walking, taking something, or closing a door are labeled.

- MuHAVI dataset [107]: By targeting silhouette-based human action recognition methods, this dataset includes video data obtained from multiple cameras. Images are taken with night street light illumination at a constant but uneven background. At each corner and each side of a rectangular platform a Schwan CCTV camera is installed. These cameras captured, according to our taxonomy, 16 different actions (WalkTurnBack, RunStop, Punch Kick, hotGunCollapse, PullHeavyObject, Pickup ThrowObject, WalkFall, LookInCar, CrawlOnKnees, WaveArms, JumpOverFence, DrunkWalk, ClimbLadder, SmashObject, JumpOverGap) and one activity (DrawGraffiti) performed by 7 actors, three times each. Each frame has a $720 \times 576$ pixels resolution and is taken at 25 fps. Nevertheless, silhouettes are annotated only at a small sub-set of the available video data.

- UCF sport action datasets [96]: Among other datasets available at UCF, this dataset stands out as it contains nearly 200 video sequences at a resolution of $720 \times 480$ pixels. Images are intentionally taken from real scenarios (usually from broadcast television channels), as on purpose recorded performances from actors lead to unrealistic and laboratory-conditioned training data. On the contrary, images taken from sport broadcasting; or from Youtube, as happens at the UCF50 dataset; present large variations in camera motion, object appearance and scale, viewpoint, clutter and illumination settings; and are therefore very challenging. Considering our taxonomy of HBA levels, this dataset does not only include actions (walking, swinging, running, diving, golf swinging, kicking, lifting), but also activities (horseback riding, skating).

- CAVIAR test scenarios: The CAVIAR project [20] also published its database. Its images are taken in two different scenarios: an entrance lobby and a shopping center. Activities of real scenarios are recorded (walking alone, meeting other people, window shopping, entering and exiting shops, fighting, passing out and leaving a package in a public place) at a resolution of $384 \times 288$ pixels. Ground-truth 
data is provided in XML format at frame level. Video sequences, taken from wide angle cameras installed as surveillance cameras at the ceiling corners, include several persons, as well as crowd movements.

- CMU-MMAC database [26]: The multi-modal activity database from the Carnegie Mellon University targets cooking and food preparation activities. Not only video data has been taken, but also audio and other sensor data (motion, accelerometers and gyroscopes). Five subjects were recorded in a kitchen while preparing five different recipes: brownies, pizza, sandwich, salad and scrambled eggs. Video images were taken from three high spatial resolution cameras $(1024 \times 768)$ at low temporal resolution (30 fps) and three low spatial cameras $(640 \times 480)$, two at high temporal resolution (60 fps), and a wearable one at low temporal resolution (12 fps). Audio data was recorded with five balanced microphones and a wearable watch. Motion was captured with 12 infrared cameras of $4 \mathrm{MP}$ at $120 \mathrm{fps}$. Five 3-axis accelerometers and gyroscopes contributed to the rest of the data. The computers used to record the sensor data were synchronised using the Network Time Protocol (NTP).

- PlaceLab datasets [42]: The PlaceLab live-in laboratory provides a full home-like environment for data gathering for ubiquitous technologies and home settings studies. Two datasets are available; whereas PLIA1 is a legacy dataset, PLIA2 improves data sharing and visualization by employing new data formats. This second dataset is also compatible with their visualization and annotation tool called Handlense. PLIA2 includes 4 hours of video data (infrared and RGB), in which one subject performs common household activities (preparing a recipe, doing a load of dishes, cleaning the kitchen, doing laundry, making the bed, and light cleaning around the apartment). Besides video data, while performing the activities, accelerometer data is recorded by so called MITes, which are attached to objects of interest (i.e. objects which are related to human activities) as remote controls, chairs, etc. Videos are annotated not only with the type of activity, but also with body posture, location and social context.

Table 2 summarises the details of the reviewed datasets. 
Table 2: Comparison of dataset features

\begin{tabular}{lccccccc}
\hline Dataset & DoS & 'Actions' & $\begin{array}{c}\text { Multi- } \\
\text { view }\end{array}$ & $\begin{array}{c}\text { Maximum } \\
\text { resolution }\end{array}$ & $\begin{array}{c}\text { Back- } \\
\text { ground }\end{array}$ & $\begin{array}{c}\text { Silhou- } \\
\text { ettes }\end{array}$ & $\begin{array}{c}\text { Out-/ } \\
\text { Indoor }\end{array}$ \\
\hline HOHA & Actions & $8 / 12$ & No & 240 lines & complex & No & both \\
KTH & Actions & 6 & No & $160 \times 120$ & simple & No & both \\
Weizmann & Actions & 10 & No & $180 \times 144$ & simple & Yes & outdoor \\
INRIA-XMAS & Actions & 13 & Yes & $390 \times 291$ & simple & Yes & indoor \\
TUM Kitchen & Actions & $10^{a}$ & Yes & $780 \times 582$ & simple & No & indoor \\
MuHAVI & both & 17 & Yes & $720 \times 576$ & complex & Yes & indoor \\
UCF Sports & both & 9 & No & $720 \times 480$ & complex & No & both \\
CAVIAR & Activities & 6 & Yes & $384 \times 288$ & complex & No & indoor \\
CMU-MMAC & Activities & 5 & Yes & $1024 \times 768$ & simple & No & indoor \\
PlaceLab (PLIA2) & Activities & 6 & Yes & $320 \times 240$ & simple & No & indoor \\
\hline
\end{tabular}

a Approximately 10 annotated sub-actions of 1 activity: setting the table.

$b$ They are provided in the Manually-Annotated Subset (MAS).

\subsection{Frameworks and Tools}

This section will detail recently appeared frameworks and tools in the field of HBA and AAL in smart homes. As these fields present a lack of standards and interoperability, first steps in multipurpose design of tools; as languages, meta-models and frameworks; have been taken in the last few years:

- Home markup language [79]: HomeML is an XML based schema for representation of information within smart homes. As data taken at a smart home scenario belongs to heterogeneous nature, and is captured by different type of sensors; this language offers an open standard for the exchange of data in a system-, application- and formatindependent way. Their ultimate goal is to support the exchange of data and to build an open data repository. HomeML supports a data structure which is designed upon the most used standards in integration of home services and devices: OSGi and KNX. This data structure is designed as a series of hierarchical data trees which enables a classified storage of the descriptions of the smart home environment (rooms, floors, inhabitants), and its devices and related events.

- ViPER - The Video Performance Evaluation Resource [50]: ViPER is a framework which targets semantic video analysis and includes several tools which make system evaluation easier. In this sense, 
the framework includes a Ground Truth Authoring Tool which incorporates a GUI to edit ground truth data and check generated metadata frame by frame. Once this step is done, performance of our recognition algorithm can be evaluated with batch-processes in a UNIX environment. In addition, a run-time application loader for JavaBeans and a Java MPEG-1 decoder with frame indexing are provided. As video metadata is stored in XML format and follows a specially designed structure, an API is provided in the form of a set of Java interfaces to access metadata programmatically; as well as a browser which visualises ground truth data and analyses results in several representation forms.

- Hong et al.'s activity meta-model: In [38], Hong et al. present a new meta-model for activity recognition in smart homes. A diagram, which is similar to an Entity-Relation, is used to build evidential networks which express the interaction between recognised activities and objects. This way, relationships between activities and objects, as well as generalization at activity level and compulsory or optional interaction with objects can be captured. Sensors' associations to objects and vice versa can be captured too. For instance, in Fig. 10, the activity of making a cold drink is associated with the composite object cup-juice. The objects cup and juice are compulsory to their combination, i.e. the composite object. Whereas the object cup is associated directly with the sensor called scup, the object juice is derived from the object fridge and this one is the object which is associated to the sensor of the fridge.

- BehaviourScope Framework [6]: The Embedded Networks and Applications Lab at the University of Yale developed a scalable framework for detailed behaviour interpretation of the elderly. Its aim is to process, communicate and present heterogeneous sensor data in an automated form, in order to infer high-level semantic data, which can be further processed at applications and services (generation of alarms, reports, triggers and answers to queries is considered). Sensors like passive infrared, door/windows opening and cameras are supported, whereas new types of sensors can be added by developing the appropriate driver for the gateway. Cameras are not used for video streaming, but for motion detection and tracking based on a motion histogram; their aim is to 


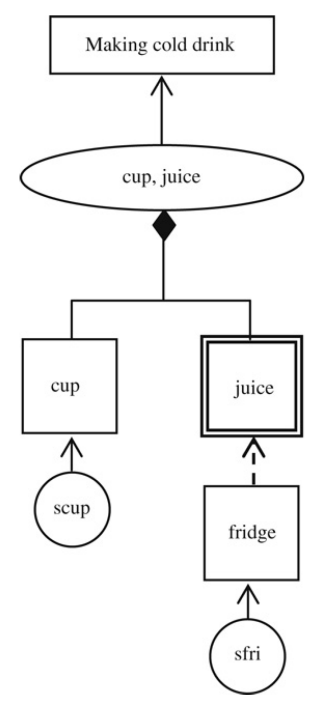

Figure 10: Evidential network representation of making a cold drink. (reprinted from [38], (C)Elsevier, 2008).

include this processing in a new camera chip, that would avoid providing any image information to the rest of the system. The framework also includes a Web portal for visualization and customization, and a mobile phone application to provide personal safety services.

- OpenAAL [126]: The FZI Research Center for IT, the FriedrichSchiller University of Jena and the CAS Software AG released this open source middleware for AAL last year. OpenAAL has been developed since 2007, as it started as the technical development of the SOPRANO Integrated Project (Sixth Framework Programme of IST) [125]. On top of the OSGi service-oriented framework, OpenAAL provides generic platform services based on three main components: 1) the Context Manager, where ambient data and information from sensors and user inputs are collected and stored supporting context reasoning at multiple levels of abstraction (from sensor and actuator states to environment characteristics); 2) The Procedural Manager which is in charge of handling installation-independent workflows which are able to react to situations of interest. These workflows are defined in BPEL with context-aware extensions in order to be able to communicate with the Context Manager; and 3) The Composer selects the available ser- 
vices in the concrete installation to achieve the abstract service goals; these are described in the installation-independent workflows. This way, abstract services can be concreted with the appropriate combination of services in order to adapt to the user's needs in each situation.

The middleware is available online with LGPL license and documentation is provided. The developed code is written in Java and uses the open source implementation of the OSGi R4 core framework specification Equinox.

\section{Conclusions}

This paper has covered the different levels of HBA following an abstraction, degree of semantics and time oriented classification. Going through recent examples of research works; most used and promising feature types, recognition methods and system design methodologies have been detailed. From pose, gaze and motion estimation to behaviour recognition, and following an initially defined classification, we have analysed vision and multimodal-based approaches.

Clearly, it can be seen that at the motion, pose and gaze estimation level, several methods achieve robust and high success rates. In conjunction with the action level, these show the most advanced and successful results. Nevertheless at higher levels, especially at behaviour, there is still a long way to go to achieve off-the-shelf products. Still, huge advances have been made in the last ten years. But the challenge to design and develop stable and general systems still persists, as most systems only solve specific problems in very particular environments.

Especially at the field of AAL, advances in this works are very valuable as personal autonomy and quality of life for elderly and cognitively impaired people can be improved enormously by these systems, and at the same time care costs can be reduced significantly.

\section{Acknowledgements.}

This work has been partially supported by the Spanish Ministry of Science and Innovation under project "Sistema de visión para la monitorización de la actividad de la vida diaria en el hogar" (TIN2010-20510-C04-02). Alexandros Andre Chaaraoui acknowledges financial support by the Conselleria d'Educació, Formació i Ocupació of the Generalitat Valenciana (fellowship 
ACIF/2011/160). The funders had no role in study design, data collection and analysis, decision to publish, or preparation of the manuscript.

\section{References}

[1] Agarwal, A., \& Triggs, B. (2004). 3d human pose from silhouettes by relevance vector regression. In Computer Vision and Pattern Recognition, 2004. CVPR 2004. Proceedings of the 2004 IEEE Computer Society Conference on (pp. II-882 - II-888). volume 2.

[2] Altun, K., \& Barshan, B. (2010). Human activity recognition using inertial/magnetic sensor units. In A. Salah, T. Gevers, N. Sebe, \& A. Vinciarelli (Eds.), Human Behavior Understanding (pp. 38-51). Springer Berlin / Heidelberg volume 6219 of Lecture Notes in Computer Science.

[3] Aminian, K., Robert, P., Buchser, E., Rutschmann, B., Hayoz, D., \& Depairon, M. (1999). Physical activity monitoring based on accelerometry: validation and comparison with video observation. Medical and Biological Engineering and Computing, 37, 304-308.

[4] Anderson, D., Luke, R., Keller, J., Skubic, M., Rantz, M., \& Aud, M. (2009). Modeling human activity from voxel person using fuzzy logic. Fuzzy Systems, IEEE Transactions on, 17, $39-49$.

[5] Andriluka, M., Roth, S., \& Schiele, B. (2009). Pictorial structures revisited: People detection and articulated pose estimation. 2009 IEEE Conference on Computer Vision and Pattern Recognition, (pp. 10141021).

[6] Bamis, A., Lymberopoulos, D., Teixeira, T., \& Savvides, A. (2010). The behaviorscope framework for enabling ambient assisted living. Personal and Ubiquitous Computing, 14, 473-487.

[7] Bandouch, J., Engstler, F., \& Beetz, M. (2008). Accurate human motion capture using an ergonomics-based anthropometric human model. In F. Perales, \& R. Fisher (Eds.), Articulated Motion and Deformable Objects (pp. 248-258). Springer Berlin / Heidelberg volume 5098 of Lecture Notes in Computer Science. 
[8] Bao, L., \& Intille, S. (2004). Activity Recognition from User-Annotated Acceleration Data. In A. Ferscha, \& F. Mattern (Eds.), Pervasive Computing (pp. 1-17). Springer Berlin / Heidelberg volume 3001 of Lecture Notes in Computer Science.

[9] Beetz, M., Bandouch, J., Jain, D., \& Tenorth, M. (2010). Towards automated models of activities of daily life. Technology and Disability, $4,1-11$.

[10] Belongie, S., Malik, J., \& Puzicha, J. (2000). Shape context: A new descriptor for shape matching and object recognition. In Proceedings of the Neural Information Processing Systems 2000 (pp. 831-837).

[11] Ben-Arie, J., Wang, Z., \& Pandit, P. (2002). Human activity recognition using multidimensional indexing. IEEE Transactions on Pattern Analysis and Machine Intelligence, 24, 1091-1104.

[12] Bobick, A., \& Davis, J. (2001). The recognition of human movement using temporal templates. Pattern Analysis and Machine Intelligence, 23, 257-267.

[13] Boulay, B., Bremond, F., \& Thonnat, M. (2006). Applying 3d human model in a posture recognition system. Pattern Recognition Letters, 27, 1788-1796.

[14] Bourdev, L., Maji, S., Brox, T., \& Malik, J. (2010). Detecting people using mutually consistent poselet activations. In Proceedings of the 11th European conference on Computer vision: Part VI ECCV'10 (pp. 168-181). Berlin, Heidelberg: Springer-Verlag.

[15] Brand, M. (1996). Coupled hidden markov models for modeling interacting processes. Neural Computation, 405, 1-28.

[16] Brdiczka, O., Crowley, J., \& Reignier, P. (2009). Learning situation models in a smart home. Systems, Man, and Cybernetics, Part B: Cybernetics, IEEE Transactions on, 39, 56-63.

[17] Canton-Ferrer, C., Segura, C., Pardas, M., Casas, J., \& Hernando, J. (2008). Multimodal real-time focus of attention estimation in smartrooms. In Computer Vision and Pattern Recognition Workshops, 2008. CVPRW '08. IEEE Computer Society Conference on (pp. 1-8). 
[18] Cardinaux, F., Brownsell, S., Hawley, M., \& Bradley, D. (2008). Modelling of behavioural patterns for abnormality detection in the context of lifestylereassurance. In J. Ruiz-Shulcloper, \& W. Kropatsch (Eds.), Progress in Pattern Recognition, Image Analysis and Applications (pp. 243-251). Springer Berlin / Heidelberg volume 5197 of Lecture Notes in Computer Science.

[19] Carranza, J., Theobalt, C., Magnor, M. A., \& Seidel, H.-P. (2003). Free-viewpoint video of human actors. In ACM SIGGRAPH 2003 Papers SIGGRAPH '03 (pp. 569-577). New York, NY, USA: ACM.

[20] CAVIAR Project (2004). Caviar test case scenarios. http://homepages.inf.ed.ac.uk/rbf/CAVIARDATA1. Last access on $18 / 01 / 2012$.

[21] Cheng, H., Yang, C., Han, F., \& Sawhney, H. (2008). Ho2: A new feature for multi-agent event detection and recognition. In Computer Vision and Pattern Recognition Workshops, 2008. CVPRW' '08. IEEE Computer Society Conference on (pp. 1-8).

[22] Cherla, S., Kulkarni, K., Kale, A., \& Ramasubramanian, V. (2008). Towards fast, view-invariant human action recognition. In Computer Vision and Pattern Recognition Workshops, 2008. CVPRW' '08. IEEE Computer Society Conference on (pp. 1 -8).

[23] Cheung, K., Baker, S., \& Kanade, T. (2003). Shape-from-silhouette of articulated objects and its use for human body kinematics estimation and motion capture. In Computer Vision and Pattern Recognition, 2003. Proceedings. 2003 IEEE Computer Society Conference on (pp. I-77 - I-84). volume 1.

[24] Chung, P.-C., \& Liu, C.-D. (2008). A daily behavior enabled hidden markov model for human behavior understanding. Pattern Recognition, 41, 1572-1580.

[25] Dalal, N., \& Triggs, B. (2005). Histograms of oriented gradients for human detection. In Proceedings of the 2005 IEEE Computer Society Conference on Computer Vision and Pattern Recognition (CVPR'05), Volume 1 CVPR '05 (pp. 886-893). Washington, DC, USA: IEEE Computer Society. 
[26] De la Torre Frade, F., Hodgins, J. K., Bargteil, A. W., Martin Artal, X., Macey, J. C., Collado I Castells, A., \& Beltran, J. (2008). Guide to the Carnegie Mellon University Multimodal Activity (CMUMMAC) Database. Technical Report CMU-RI-TR-08-22 Robotics Institute Pittsburgh, PA.

[27] Dempster, A. P. (1968). A generalization of bayesian inference. Journal of the Royal Statistical Society. Series B (Methodological), 30, 205-247.

[28] Doshi, A., \& Trivedi, M. (2010). Attention estimation by simultaneous observation of viewer and view. In Computer Vision and Pattern Recognition Workshops (CVPRW), 2010 IEEE Computer Society Conference on (pp. 21-27). IEEE.

[29] Doshi, A., \& Trivedi, M. (2010). Examining the impact of driving style on the predictability and responsiveness of the driver: Real-world and simulator analysis. In Intelligent Vehicles Symposium (IV), 2010 IEEE (pp. 232-237). IEEE.

[30] Duong, T., Bui, H., Phung, D., \& Venkatesh, S. (2005). Activity recognition and abnormality detection with the switching hidden semimarkov model. In Computer Vision and Pattern Recognition, 2005. CVPR 2005. IEEE Computer Society Conference on (pp. 838-845). volume 1.

[31] Eichner, M., \& Ferrari, V. (2009). Better appearance models for pictorial structures. In Proceedings of the British Machine Vision Conference (pp. 1-11).

[32] Felzenszwalb, P., Girshick, R., McAllester, D., \& Ramanan, D. (2010). Object detection with discriminatively trained part-based models. Pattern Analysis and Machine Intelligence, IEEE Transactions on, 32, $1627-1645$.

[33] Ferrari, V., Marin-Jimenez, M., \& Zisserman, A. (2008). Progressive search space reduction for human pose estimation. In Computer Vision and Pattern Recognition, 2008. CVPR 2008. IEEE Conference on (pp. $1-8)$.

[34] Gavrila, D. (1999). The visual analysis of human movement: A survey. Computer Vision and Image Understanding, 73, 82-98. 
[35] Gorelick, L., Blank, M., Shechtman, E., Irani, M., \& Basri, R. (2007). Actions as space-time shapes. Transactions on Pattern Analysis and Machine Intelligence, 29, 2247-2253.

[36] Hara, K., Omori, T., \& Ueno, R. (2002). Detection of unusual human behavior in intelligent house. In Neural Networks for Signal Processing, 2002. Proceedings of the 2002 12th IEEE Workshop on (pp. 697 - 706).

[37] Hayes, T., Pavel, M., \& Kaye, J. (2008). An approach for deriving continuous health assessment indicators from in-home sensor data. In Technology and Aging: Selected Papers from the 2007 International Conference on Technology and Aging (pp. 130-137). Ios Press volume 21 .

[38] Hong, X., Nugent, C., Mulvenna, M., McClean, S., Scotney, B., \& Devlin, S. (2009). Evidential fusion of sensor data for activity recognition in smart homes. Pervasive and Mobile Computing, 5, 236-252.

[39] Hongeng, S., \& Nevatia, R. (2001). Multi-agent event recognition. In Computer Vision, 2001. ICCV 2001. Proceedings. Eighth IEEE International Conference on (pp. 84-91). IEEE volume 2.

[40] Howe, N. (2004). Silhouette lookup for automatic pose tracking. In Computer Vision and Pattern Recognition Workshop, 2004. CVPRW '04. Conference on (pp. 15-22).

[41] Hu, W., Tan, T., Wang, L., \& Maybank, S. (2004). A survey on visual surveillance of object motion and behaviors. IEEE Transactions on Systems, Man and Cybernetics, Part C (Applications and Reviews), 34, 334-352.

[42] Intille, S. S., Larson, K., Tapia, E. M., Beaudin, J., Kaushik, P., Nawyn, J., \& Rockinson, R. (2006). Using a live-in laboratory for ubiquitous computing research. In Proceedings of PERVASIVE06 (pp. 349-365).

[43] Jaimes, A., \& Sebe, N. (2007). Multimodal humancomputer interaction: A survey. Computer Vision and Image Understanding, 108, $116-134$. 
[44] Jain, G., Cook, D., \& Jakkula, V. (2006). Monitoring health by detecting drifts and outliers for a smart environment inhabitant. In Proceedings of the International Conference On Smart Homes and Health Telematics (pp. 1-8).

[45] Ji, X., Liu, H., Li, Y., \& Brown, D. (2008). Visual-based view-invariant human motion analysis: A review. In I. Lovrek, R. Howlett, \& L. Jain (Eds.), Knowledge-Based Intelligent Information and Engineering Systems (pp. 741-748). Springer Berlin / Heidelberg volume 5177 of Lecture Notes in Computer Science.

[46] Juan, L., \& Gwon, O. (2009). A Comparison of SIFT, PCA-SIFT and SURF. International Journal of Image Processing (IJIP), 3, 143-152.

[47] Kadir, T., \& Brady, M. (2003). Scale saliency: A novel approach to salient feature and scale selection. In Visual Information Engineering, 2003. VIE 2003. International Conference on (pp. 25-28). IET.

[48] Karaman, S., Benois-Pineau, J., Mégret, R., Dovgalecs, V., Dartigues, J., \& Gäestel, Y. (2010). Human daily activities indexing in videos from wearable cameras for monitoring of patients with dementia diseases. In 2010 International Conference on Pattern Recognition (pp. 41134116). IEEE.

[49] Kwapisz, J., Weiss, G., \& Moore, S. (2011). Activity recognition using cell phone accelerometers. ACM SIGKDD Explorations Newsletter, 12, $74-82$.

[50] Language and Media Processing Laboratory, University of Maryland (2003). Viper: The video performance evaluation resource. http://viper-toolkit.sourceforge.net. Last access on 18/01/2012.

[51] Laptev, I. (2005). On space-time interest points. International Journal of Computer Vision, 64, 107-123.

[52] Laptev, I., Marszalek, M., Schmid, C., \& Rozenfeld, B. (2008). Learning realistic human actions from movies. In Computer Vision and Pattern Recognition, 2008. CVPR 2008. IEEE Conference on (pp. 1 -8). 
[53] Launila, A., \& Sullivan, J. (2010). Contextual features for head pose estimation in football games. In Pattern Recognition (ICPR), 2010 20th International Conference on (pp. 340 -343).

[54] Lester, J., Choudhury, T., \& Borriello, G. (2006). A practical approach to recognizing physical activities. In Proceedings of Pervasive (pp. 1$16)$.

[55] Li, W., Zhang, Z., \& Liu, Z. (2010). Action recognition based on a bag of 3d points. In Computer Vision and Pattern Recognition Workshops (CVPRW), 2010 IEEE Computer Society Conference on (pp. 9 -14).

[56] Li, W., Zhang, Z., Liu, Z., \& Member, S. (2008). Expandable datadriven graphical modeling of human actions based on salient postures. IEEE Transactions on Circuits and Systems for Video Technology, 18, $1499-1510$.

[57] Liu, C.-D., Chung, Y.-N., \& Chung, P.-C. (2010). An interactionembedded hmm framework for human behavior understanding: With nursing environments as examples. Information Technology in Biomedicine, IEEE Transactions on, 14, 1236-1246.

[58] Lowe, D. G. (2004). Distinctive image features from scale-invariant keypoints. International Journal of Computer Vision, 60, 91-110.

[59] Lucas, B. D. (1981). An iterative image registration technique with an application to stereo vision. Imaging, 130, 121-129.

[60] Lv, F., \& Nevatia, R. (2007). Single view human action recognition using key pose matching and viterbi path searching. In Computer Vision and Pattern Recognition, 200\%. CVPR '07. IEEE Conference on (pp. $1-8$ ).

[61] Mahmoud, S., Akhlaghinia, M., Lotfi, A., \& Langensiepen, C. (2011). Trend modelling of elderly lifestyle within an occupancy simulator. In Computer Modelling and Simulation (UKSim), 2011 UkSim 13th International Conference on (pp. 156 -161).

[62] Marin-Jimenez, M., Zisserman, A., \& Ferrari, V. (2011). "Here's looking at you, kid." Detecting people looking at each other in videos. In Proceedings of the British Machine Vision Conference. 
[63] Maurer, U., Smailagic, A., Siewiorek, D., \& Deisher, M. (2006). Activity recognition and monitoring using multiple sensors on different body positions. In Wearable and Implantable Body Sensor Networks, 2006. BSN 2006. International Workshop on (pp. 113-116).

[64] Mcivor, A. M. (2000). Background Subtraction Techniques. In Proceedings of Image and Vision Computing, Auckland, New Zealand, 2000.

[65] Messing, R., Pal, C., \& Kautz, H. (2009). Activity recognition using the velocity histories of tracked keypoints. In Computer Vision, 2009 IEEE 12th International Conference on (pp. 104-111).

[66] van der Meulen, P., \& Seidl, A. (2007). Ramsis the leading cad tool for ergonomic analysis of vehicles. In V. Duffy (Ed.), Digital Human Modeling (pp. 1008-1017). Springer Berlin / Heidelberg volume 4561 of Lecture Notes in Computer Science.

[67] Mihailidis, A., Boger, J. N., Craig, T., \& Hoey, J. (2008). The coach prompting system to assist older adults with dementia through handwashing: an efficacy study. BMC geriatrics, 8, 28.

[68] Mihailidis, A., Carmichael, B., \& Boger, J. (2004). The use of computer vision in an intelligent environment to support aging-in-place, safety, and independence in the home. Information Technology in Biomedicine, IEEE Transactions on, 8, 238 -247.

[69] Mihailidis, A., Fernie, G. R., \& Cleghorn, W. L. (2000). The development of a computerized cueing device to help people with dementia to be more independent. Technology and Disability, 13, 23-40.

[70] Mikić, I., Trivedi, M., Hunter, E., \& Cosman, P. (2003). Human body model acquisition and tracking using voxel data. International Journal of Computer Vision, 53, 199-223.

[71] Moeslund, T. (2001). A survey of computer vision-based human motion capture. Computer Vision and Image Understanding, 81, 231-268.

[72] Moeslund, T. B., Hilton, A., \& Krüger, V. (2006). A survey of advances in vision-based human motion capture and analysis. Computer Vision and Image Understanding, 104, 90-126. 
[73] Monekosso, D. N., \& Remagnino, P. (2010). Behavior analysis for assisted living. IEEE Transactions on Automation Science and Engineering, 7, 879-886.

[74] Nait-Charif, H., \& McKenna, S. (2004). Activity summarisation and fall detection in a supportive home environment. In Pattern Recognition, 2004. ICPR 2004. Proceedings of the 17th International Conference on (pp. 323-326). volume 4.

[75] Najafi, B., Aminian, K., Paraschiv-Ionescu, A., Loew, F., Büla, C. J., \& Robert, P. (2003). Ambulatory system for human motion analysis using a kinematic sensor: monitoring of daily physical activity in the elderly. IEEE transactions on bio-medical engineering, 50, 711-723.

[76] Nguyen, N., Phung, D., Venkatesh, S., \& Bui, H. (2005). Learning and detecting activities from movement trajectories using the hierarchical hidden markov model. In Computer Vision and Pattern Recognition, 2005. CVPR 2005. IEEE Computer Society Conference on (pp. 955960). volume 2 .

[77] Nicolini, C., Lepri, B., Teso, S., \& Passerini, A. (2010). From ongoing to complete activity recognition exploiting related activities. In A. Salah, T. Gevers, N. Sebe, \& A. Vinciarelli (Eds.), Human Behavior Understanding (pp. 26-37). Springer Berlin / Heidelberg volume 6219 of Lecture Notes in Computer Science.

[78] Niebles, J. C., Wang, H., \& Fei-Fei, L. (2008). Unsupervised learning of human action categories using spatial-temporal words. International Journal of Computer Vision, 79, 299-318.

[79] Nugent, C. D., Finlay, D. D., Davies, R. J., Wang, H. Y., Zheng, H., Hallberg, J., Synnes, K., \& Mulvenna, M. D. (2007). homeml: an open standard for the exchange of data within smart environments. In Proceedings of the 5th international conference on Smart homes and health telematics ICOST'07 (pp. 121-129). Berlin, Heidelberg: Springer-Verlag.

[80] Oikonomopoulos, A., Pantic, M., \& Patras, I. (2008). B-spline polynomial descriptors for human activity recognition. In Computer Vision 
and Pattern Recognition Workshops, 2008. CVPRW'08. IEEE Computer Society Conference on (pp. $1-6)$.

[81] Oikonomopoulos, A., Patras, I., \& Pantic, M. (2005). Spatiotemporal salient points for visual recognition of human actions. Systems, Man, and Cybernetics, Part B: Cybernetics, IEEE Transactions on, 36, 710 -719 .

[82] Oikonomopoulos, A., Patras, I., \& Pantic, M. (2009). An implicit spatiotemporal shape model for human activity localization and recognition. 2009 IEEE Computer Society Conference on Computer Vision and Pattern Recognition Workshops, (pp. 27-33).

[83] Oliver, N. M., Rosario, B., \& Pentland, A. P. (2000). A bayesian computer vision system for modeling human interactions. IEEE Transactions on Pattern Analysis and Machine Intelligence, 22, 831-843.

[84] Ozturk, O., Yamasaki, T., \& Aizawa, K. (2009). Tracking of humans and estimation of body/head orientation from top-view single camera for visual focus of attention analysis. In Computer Vision Workshops (ICCV Workshops), 2009 IEEE 12th International Conference on (pp. 1020-1027).

[85] Park, K., Lin, Y., Metsis, V., Le, Z., \& Makedon, F. (2010). Abnormal human behavioral pattern detection in assisted living environments. In Proceedings of the 3rd International Conference on PErvasive Technologies Related to Assistive Environments PETRA '10 (pp. 9:1-9:8). New York, NY, USA: ACM.

[86] Park, S., \& Kautz, H. (2008). Privacy-preserving recognition of activities in daily living from multi-view silhouettes and rfid-based training. In AAAI Fall 2008 Symposium on AI in Eldercare: New Solutions to Old Problems.

[87] Poppe, R. (2007). Vision-based human motion analysis: An overview. Computer Vision and Image Understanding, 108, 4-18.

[88] Poppe, R. (2010). A survey on vision-based human action recognition. Image and Vision Computing, 28, 976-990. 
[89] Porle, R. R., Chekima, A., Wong, F., \& Sainarayanan, G. (2009). Performance of histogram-based skin colour segmentation for arms detection in human motion analysis application. International Journal of Electronics, Communications and Computer Engineering, 1, 403-408.

[90] Quintas, J., Khoshhal, K., Aliakbarpour, H., Hofmann, M., \& Dias, J. (2011). Using concurrent hidden markov models to analyse human behaviours in a smart home environment. In 12th International Workshop on Image Analysis for Multimedia Interactive Services.

[91] Reale, M., Hung, T., \& Yin, L. (2010). Pointing with the eyes: Gaze estimation using a static/active camera system and $3 \mathrm{~d}$ iris disk model. In Multimedia and Expo (ICME), 2010 IEEE International Conference on (pp. 280-285). IEEE.

[92] Reale, M., Hung, T., \& Yin, L. (2010). Viewing direction estimation based on 3d eyeball construction for hri. In Computer Vision and Pattern Recognition Workshops (CVPRW), 2010 IEEE Computer Society Conference on (pp. 24-31). IEEE.

[93] Ren, X., \& Philipose, M. (2009). Egocentric recognition of handled objects: Benchmark and analysis. In IEEE Computer Society Conference on Computer Vision and Pattern Recognition Workshops (pp. 1-8). IEEE.

[94] Robertson, N., \& Reid, I. (2006). A general method for human activity recognition in video. Computer Vision and Image Understanding, 104, $232-248$.

[95] Robertson, N., Reid, I., \& Brady, M. (2006). Behaviour recognition and explanation for video surveillance. In Crime and Security, 2006. The Institution of Engineering and Technology Conference on (pp. 458463).

[96] Rodriguez, M., Ahmed, J., \& Shah, M. (2008). Action mach a spatiotemporal maximum average correlation height filter for action recognition. In Computer Vision and Pattern Recognition, 2008. CVPR 2008. IEEE Conference on (pp. 1-8). 
[97] Roetenberg, D., Slycke, P. J., \& Veltink, P. H. (2007). Ambulatory position and orientation tracking fusing magnetic and inertial sensing. IEEE transactions on bio-medical engineering, 54, 883-90.

[98] Rosales, R., \& Sclaroff, S. (2000). Learning and synthesizing human body motion and posture. In Automatic Face and Gesture Recognition, 2000. Proceedings. Fourth IEEE International Conference on (pp. 506 $-511)$.

[99] Rybok, L., Voit, M., Ekenel, H., \& Stiefelhagen, R. (2010). Multiview based estimation of human upper-body orientation. In Pattern Recognition (ICPR), 2010 20th International Conference on (pp. 15581561).

[100] Ryoo, M., \& Aggarwal, J. (2009). Spatio-temporal relationship match: Video structure comparison for recognition of complex human activities. In Computer Vision, 2009 IEEE 12th International Conference on (pp. $1593-1600)$.

[101] Sapp, B., Toshev, A., \& Taskar, B. (2010). Cascaded models for articulated pose estimation. In Proceedings of the 11th European conference on Computer vision: Part II ECCV'10 (pp. 406-420). Berlin, Heidelberg: Springer-Verlag.

[102] Schuldt, C., Laptev, I., \& Caputo, B. (2004). Recognizing human actions: a local svm approach. In Pattern Recognition, 2004. ICPR 2004. Proceedings of the 17th International Conference on (pp. 32-36). volume 3.

[103] Shafer, G. (1976). A mathematical theory of evidence. Princeton University Press.

[104] Shakhnarovich, G., Viola, P., \& Darrell, T. (2003). Fast pose estimation with parameter-sensitive hashing. In Computer Vision, 2003. Proceedings. Ninth IEEE International Conference on (pp. 750-757). volume 2.

[105] Shimizu, H., \& Poggio, T. (2003). Direction estimation of pedestrian from images. 
[106] Shotton, J., Fitzgibbon, A. W., Cook, M., Sharp, T., Finocchio, M., Moore, R., Kipman, A., \& Blake, A. (2011). Real-time human pose recognition in parts from single depth images. In Proceedings of the 24th IEEE Conference on Computer Vision and Pattern Recognition, CVPR 2011 (pp. 1297-1304).

[107] Singh, S., Velastin, S., \& Ragheb, H. (2010). Muhavi: A multicamera human action video dataset for the evaluation of action recognition methods. In Advanced Video and Signal Based Surveillance (AVSS), 2010 Seventh IEEE International Conference on (pp. 48-55). IEEE.

[108] Sminchisescu, C., Kanaujia, A., Li, Z., \& Metaxas, D. (2005). Discriminative density propagation for $3 \mathrm{~d}$ human motion estimation. In Computer Vision and Pattern Recognition, 2005. CVPR 2005. IEEE Computer Society Conference on (pp. 390-397). volume 1.

[109] Spriggs, E., De La Torre, F., \& Hebert, M. (2009). Temporal segmentation and activity classification from first-person sensing. In Computer Vision and Pattern Recognition Workshops, 2009. CVPR Workshops 2009. IEEE Computer Society Conference on (pp. 17-24).

[110] Stauffer, C., \& Grimson, W. (1999). Adaptive background mixture models for real-time tracking. In Computer Vision and Pattern Recognition, 1999. IEEE Computer Society Conference on. (pp. 246-252). volume 2.

[111] Sun, L., Klank, U., \& Beetz, M. (2009). Eyewatchme3d hand and object tracking for inside out activity analysis. In Computer Vision and Pattern Recognition Workshops, 2009. CVPR Workshops 2009. IEEE Computer Society Conference on (pp. 9-16). IEEE.

[112] Sundaram, S., \& Cuevas, W. (2009). High level activity recognition using low resolution wearable vision. In Computer Vision and Pattern Recognition Workshops, 2009. CVPR Workshops 2009. IEEE Computer Society Conference on (pp. $25-32$ ).

[113] Sutton, C., \& McCallum, A. (2007). An Introduction to Conditional Random Fields for Relational Learning. In L. Getoor, \& B. Taskar (Eds.), Introduction to Statistical Relational Learning. MIT Press. 
[114] Tao, Y., Hu, H., \& Zhou, H. (2007). Integration of vision and inertial sensors for $3 \mathrm{~d}$ arm motion tracking in home-based rehabilitation. The International Journal of Robotics Research, 26, 607-624.

[115] Tapia, E., Intille, S., \& Larson, K. (2004). Activity Recognition in the Home Using Simple and Ubiquitous Sensors Pervasive Computing. In A. Ferscha, \& F. Mattern (Eds.), Pervasive Computing chapter 10. (pp. 158-175). Berlin, Heidelberg: Springer Berlin / Heidelberg volume 3001 of Lecture Notes in Computer Science.

[116] Tenorth, M., Bandouch, J., \& Beetz, M. (2009). The tum kitchen data set of everyday manipulation activities for motion tracking and action recognition. In Computer Vision Workshops (ICCV Workshops), 2009 IEEE 12th International Conference on (pp. 1089 -1096).

[117] Toyama, K., Krumm, J., Brumitt, B., \& Meyers, B. (1999). Wallflower: principles and practice of background maintenance. In Computer Vision, 1999. The Proceedings of the Seventh IEEE International Conference on (pp. 255-261). volume 1.

[118] Turaga, P., Chellappa, R., Subrahmanian, V., \& Udrea, O. (2008). Machine recognition of human activities: A survey. IEEE Transactions on Circuits and Systems for Video Technology, 18, 1473-1488.

[119] Tuytelaars, T., \& Mikolajczyk, K. (2007). Local invariant feature detectors: A survey. Foundations and Trends in Computer Graphics and Vision, 3, 177-280.

[120] Virone, G., \& Sixsmith, A. (2008). Monitoring activity patterns and trends of older adults. In Engineering in Medicine and Biology Society, 2008. EMBS 2008. 30th Annual International Conference of the IEEE (pp. 2071-2074).

[121] Wang, L. (2003). Recent developments in human motion analysis. Pattern Recognition, 36, 585-601.

[122] Weinland, D., Boyer, E., \& Ronfard, R. (2007). Action recognition from arbitrary views using $3 \mathrm{~d}$ exemplars. In Computer Vision, $200 \%$. ICCV 2007. IEEE 11th International Conference on (pp. 1-7). 
[123] Weinland, D., Ronfard, R., \& Boyer, E. (2006). Free viewpoint action recognition using motion history volumes. Computer Vision and Image Understanding, 104, 249-257.

[124] Winn, J., Criminisi, A., \& Minka, T. (2005). Object categorization by learned universal visual dictionary. In Computer Vision, 2005. ICCV 2005. Tenth IEEE International Conference on (pp. 1800-1807). volume 2 .

[125] Wolf, P., Schmidt, A., \& Klein, M. (2008). Soprano - an extensible, open aal platform for elderly people based on semantical contracts. In 3rd Workshop on Artificial Intelligence Techniques for Ambient Intelligence (AITAmI08), 18th European Conference on Artificial Intelligence (ECAI 08), Patras, Greece.

[126] Wolf, P., Schmidt, A., Otte, J. P., Klein, M., Rollwage, S., Knig-Ries, B., Dettborn, T., \& Gabdulkhakova, A. (2010). openaal - the open source middleware for ambient-assisted living (aal). In AALIANCE conference, Malaga, Spain, March 11-12, 2010.

[127] Wood, A., Stankovic, J., Virone, G., Selavo, L., He, Z., Cao, Q., Doan, T., Wu, Y., Fang, L., \& Stoleru, R. (2008). Context-aware wireless sensor networks for assisted living and residential monitoring. IEEE Network, 22, 26-33.

[128] Wu, B., \& Nevatia, R. (2005). Detection of multiple, partially occluded humans in a single image by bayesian combination of edgelet part detectors. In Computer Vision, 2005. ICCV 2005. Tenth IEEE International Conference on (pp. 90-97). volume 1.

[129] Wu, J., Osuntogun, A., Choudhury, T., Philipose, M., \& Rehg, J. (2007). A scalable approach to activity recognition based on object use. In Computer Vision, 200\%. ICCV 200\%. IEEE 11th International Conference on (pp. 1-8). IEEE.

[130] Xu, C., \& Prince, J. (1998). Snakes, shapes, and gradient vector flow. Image Processing, IEEE Transactions on, 7, 359-369.

[131] Yamamoto, M., Mitomi, H., Fujiwara, F., \& Sato, T. (2006). Bayesian classification of task-oriented actions based on stochastic context-free 
grammar. In Automatic Face and Gesture Recognition, 2006. FGR 2006. 7th International Conference on (pp. 317 -322).

[132] Yang, A., Iyengar, S., Sastry, S., Bajcsy, R., Kuryloski, P., \& Jafari, R. (2008). Distributed segmentation and classification of human actions using a wearable motion sensor network. In Computer Vision and Pattern Recognition Workshops, 2008. CVPRW '08. IEEE Computer Society Conference on (pp. 1 -8).

[133] Zhou, Z., Chen, X., Chung, Y.-c., He, Z., Han, T. X., \& Keller, J. M. (2008). Activity analysis, summarization, and visualization for indoor human activity monitoring. IEEE Transactions on Circuits and Systems for Video Technology, 18, 1489-1498.

[134] Zouba, N., Boulay, B., Bremond, F., \& Thonnat, M. (2008). Cognitive vision. chapter Monitoring Activities of Daily Living (ADLs) of Elderly Based on 3D Key Human Postures. (pp. 37-50). Berlin, Heidelberg: Springer-Verlag. 